(C) 2006 The Japan Society of Applied Physics

\title{
Observation and Analysis of Tunneling Properties of Single Spherical Nanocrystalline Silicon Quantum Dot
}

\author{
Akhmadi SurawiJaYA $^{1 *}$, Hiroshi MizUta ${ }^{1,2}$ and Shunri ODA ${ }^{1,2}$ \\ ${ }^{1}$ Department of Physical Electronics and Quantum Nanoelectronics Research Center, Tokyo Institute of Technology, \\ 2-12-1 O-okayama, Meguro-ku, Tokyo 152-8552, Japan \\ ${ }^{2}$ SORST JST (Japan Science and Technology), 4-1-8 Honcho, Kawaguchi, Saitama 332-0012, Japan
}

(Received September 15, 2005; revised January 13, 2006; accepted January 20, 2006; published online April 25, 2006)

Electron tunneling is investigated experimentally and theoretically for a single nanocrystalline silicon quantum dot with a diameter of less than $10 \mathrm{~nm}$. Nanocrystalline silicon quantum dots are deposited by very high frequency (VHF) plasma chemical vapor deposition (CVD) sparsely over the oxide-covered silicon substrate. The dots are then naturally oxidized at room temperature by exposing it to ambient air, resulting in an ultrathin oxide tunnel barrier. A current peak is clearly observed at room temperature with the highest peak-to-valley current ratio of about 17 by contact-mode atomic force microscopy (AFM). We also perform three-dimensional numerical simulations of the transmission spectrum and tunneling current based on the scattering matrix theory. In the current-voltage $(I-V)$ characteristics calculated at room temperature we demonstrate the resonant tunneling current peaks associated with the quasi-bound state formed in a spherical silicon quantum dot.

[DOI: $10.1143 / \mathrm{JJAP} .45 .3638$ ]

KEYWORDS: resonant tunneling, room-temperature operation, nanocrystalline silicon quantum dot, atomic force microscopy, three-dimensional simulation, scattering matrix

\section{Introduction}

Resonant tunneling (RT) was first observed by Tsu and Esaki in 1974 for compound semiconductor superlattice structures. ${ }^{1)}$ RT in silicon has also been a main topic of research in the past few years mainly because of the compatibility with current IC fabrication technology. Silicon-based resonant tunneling devices (RTDs) would have a significant impact on complementary metal-oxidesemiconductor (CMOS) technology. RTDs could be integrated together with a field-effect transistor to fabricate functional devices such as low-power-tunneling-based static random-access memory (TSRAM), ${ }^{2,3)}$ and multivalued circuits. ${ }^{4)}$

Silicon on insulator (SOI) ${ }^{5)}$ was first used for studying RT in $\mathrm{Si}$, and negative differential conductance (NDC) was observed at low temperature. RT through a two-dimensionally confined structure has also been reported recently using a nanocrystalline silicon quantum disc, ${ }^{6}$ and NDC has been observed at room temperature. Quantum-dot-based IC technology is predicted to become the most basic component of ultra-scaled semiconductor devices. ${ }^{7)}$ Nanocrystallinebased RTDs can be used for local refresh in dynamic random-access memory (DRAM) with extremely low power dissipation. $^{8)}$

In this work we used a spherical nanocrystalline silicon quantum dot (nc-Si QD) to fabricate an ideal zero-dimensional RT structure. The nc-Si QD is basically a zerodimensional system with a diameter of less than $10 \mathrm{~nm}$, and quantum confinement occurs spherically. By using nanocrystalline silicons we expected room-temperature negative differential conductance (NDC) characteristics with a large peak-to-valley current ratio. In addition, we performed a three-dimensional (3D) scattering matrix simulation for a zero-dimensional RT structure ${ }^{9)}$ to analyze the transmission properties of a nanoscale Si QDs.

*E-mail address: akhmadi@neo.pe.titech.ac.jp

\section{Experimental}

For the experiment we prepared an N-type $\langle 100\rangle$ silicon substrate with a resistance of $1 \times 10^{-3}-2 \times 10^{-2} \Omega \mathrm{cm}$. A 1.5-nm-thick oxide layer was first grown on the substrate using $\mathrm{H}_{2} \mathrm{SO}_{4}: \mathrm{H}_{2} \mathrm{O}_{2}(30: 70)$ solution at $130^{\circ} \mathrm{C}$ for $10 \mathrm{~min}$. Nanocrystalline $\mathrm{Si}$ QDs were then deposited using an ultrahigh-vacuum plasma-enhanced chemical vapor deposition (UHV-PEVCD) system. The sample was then exposed to ambient air for $1-2 \mathrm{~h}$ at room temperature to oxidize the nc-Si dots naturally, forming oxide of around $0.5-1.5 \mathrm{~nm}$ thickness. Nanocrystalline Si QDs were observed by using secanning electron microscopy (SEM), and the average diameter of the nc-Si dots was found to be about $8-10 \mathrm{~nm}$ with a density of $1 \times 10^{14} \mathrm{~cm}^{-2}$. SEM image of the nc-Si dots is shown in Fig. 1. The sample was then prepared for contact-mode AFM measurement by attaching the sample to a metal disc using silver paste. For electrical characterizations we used a Au-coated contact-mode AFM tip. The

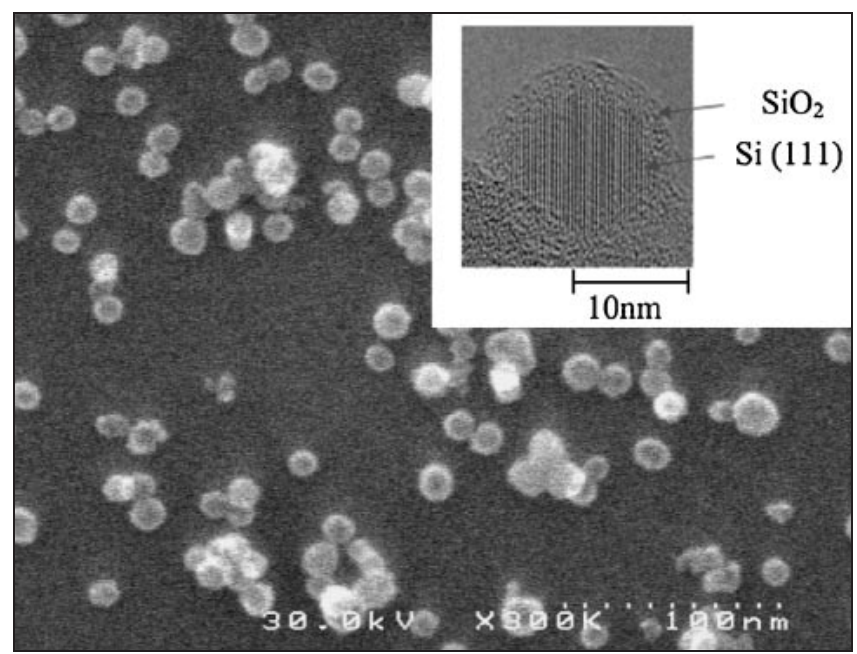

Fig. 1. SEM image of nc-Si dot deposited on chemically grown $\mathrm{SiO}_{2}$ film. The inset shows the HRTEM image of a single nc-Si QD. 


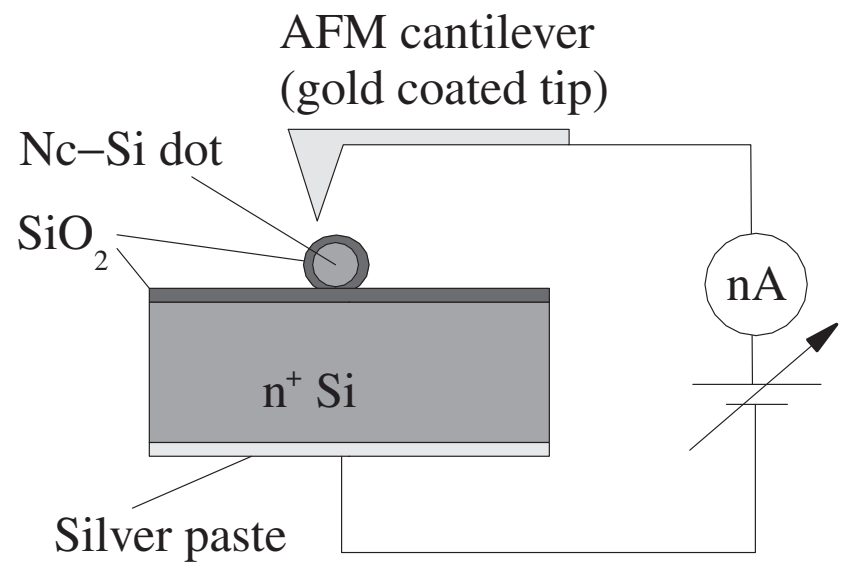

Fig. 2. Measurement setup for nc-Si QD using contact-mode AFM.

measurement setup is shown in Fig. 2. An area of $1 \times 1 \mu \mathrm{m}^{2}$ on the sample was first scanned to produce the topographical image: we then made the cantilever tip approach a dot isolated from others with an interdot distance of around $20 \mathrm{~nm}$. Topographical scanning must be repeated prior to current measurement due to drift in the AFM image.

\section{Results and Discussion}

Current-voltage $(I-V)$ characteristics are shown in Fig. 3, obtained when the tip was positively biased with respect to the substrate at room temperature. Figure 3(a) shows the $I-V$ characteristic of a sample without the nc-Si QD as a reference. It shows a monotonous increase in the background current. Figures 3(b) and 3(c) show the $I-V$ characteristics observed for two different nc-Si QDs. Current peaks were clearly observed at room temperature. In Fig. 3(b), the peakto-valley $(\mathrm{P} / \mathrm{V})$ current ratio is around 17 for the first peak. The physical origin of the current peaks will be discussed later. The characteristics were reproduced several times before thermal and current noise became dominant due to the surface contamination of the nc-Si by the AFM tip. The characteristic in Fig. 3(c) also shows the current peaks, but their peak voltages are larger than those in Fig. 3(b) and the peak currents are smaller. Both peak voltages and peak currents were found to vary to some extent depending on the $\mathrm{nc}-\mathrm{Si}$ dots chosen for measurement. One of the possible mechanisms that contribute to this fluctuation is the perturbation induced by the AFM tip to the nc-Si QD system. ${ }^{10)}$ The AFM tip is probably not always positioned right on top of the nc-Si QD, and the tip-to-dot contact resistance may vary depending on the tip position relative to the dot, affecting the peak voltage. Another possible mechanism is the different charge storage in the nc-Si QD, which increases the energy level in the QD for every electron trapped in the dot. In fact, we have observed the current hysteresis for the forward and reverse bias sweeps, which are associated with charge storage in the QD.

For studying the tunneling process via a single nc-Si QD theoretically, we also performed 3D scattering matrix simulation. ${ }^{9}{ }^{9}$ The simulation is based on the 3D Schrödinger equations with scattering boundary conditions, which are numerically solved to calculate a multimode resonant tunneling characteristic. The $3 \mathrm{D}$ device structure used for

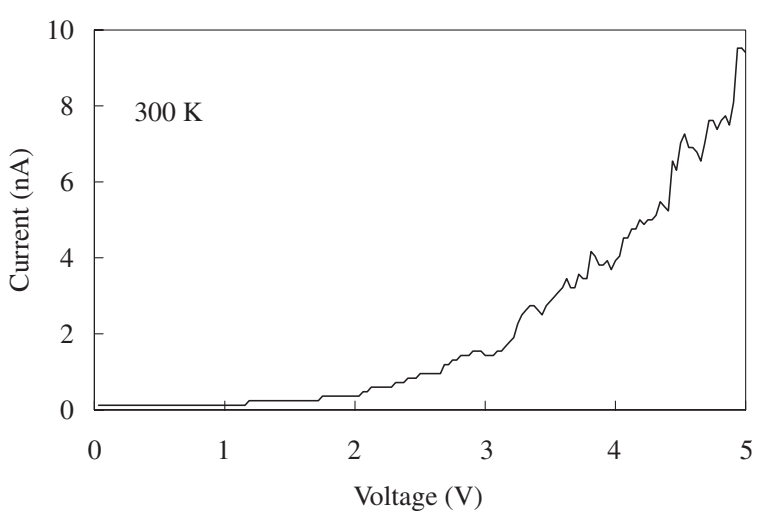

(a)

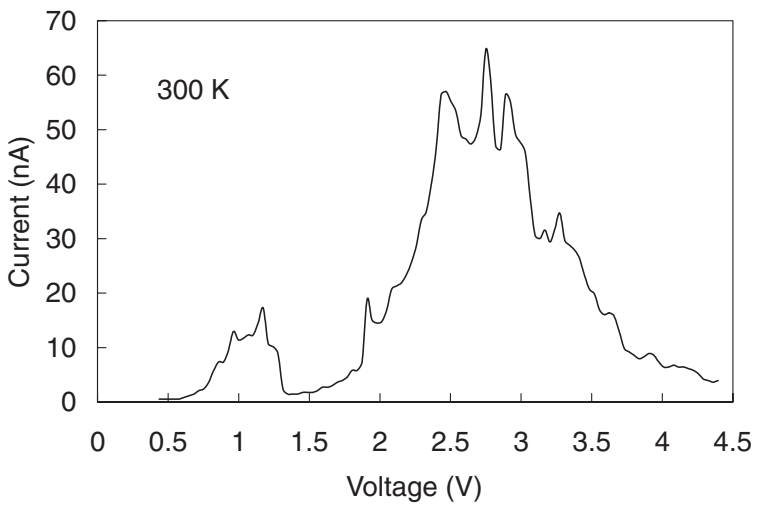

(b)

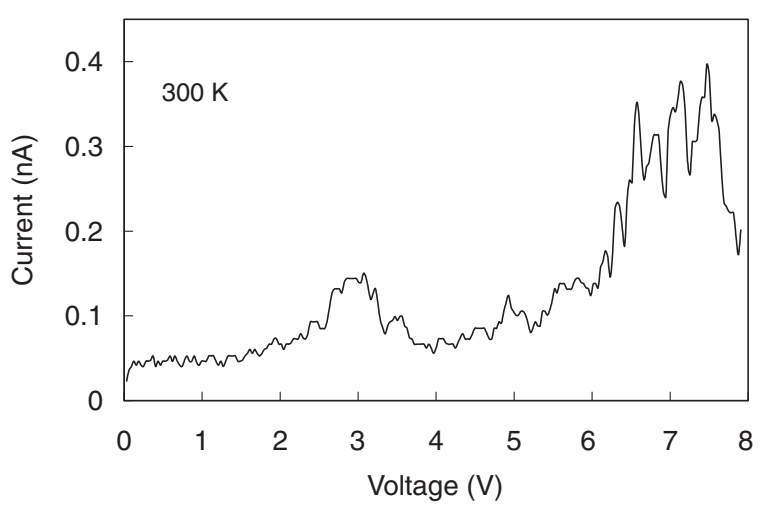

(c)

Fig. 3. Room-temperature $I-V$ characteristics. (a) $I-V$ characteristic from sample without nc-Si QD. (b). $I-V$ characteristics observed for sample with nc-Si QD. (c) $I-V$ characteristic observed for different nc-Si dot.

simulation is shown in Fig. 4. An undoped $\mathrm{Si} Q \mathrm{QD}$ is embedded in the $\mathrm{SiO}_{2}$ matrix, which is sandwiched between heavily doped $\mathrm{Si}$ emitter and collector contact regions. We assumed for the numerical simulation that the oxide barrier thickness is $0.5 \mathrm{~nm}$ and the diameter of the sphere is $7 \mathrm{~nm}$, which are consistent with the size of the $8 \mathrm{~nm}$ nc-Si QD after natural room-temperature oxidation. The transmission spectrum calculated for the device is shown in Fig. 5 with six transmission peaks indicated with roman numerals (I-VI). Two transmission subpeaks (IV and VI) were observed between the main peaks. These subpeaks are a unique feature of the spherical QD that causes a lateral-mode mixing of electron waves during the tunneling through the QD. The 3D probability density of electrons for the first 


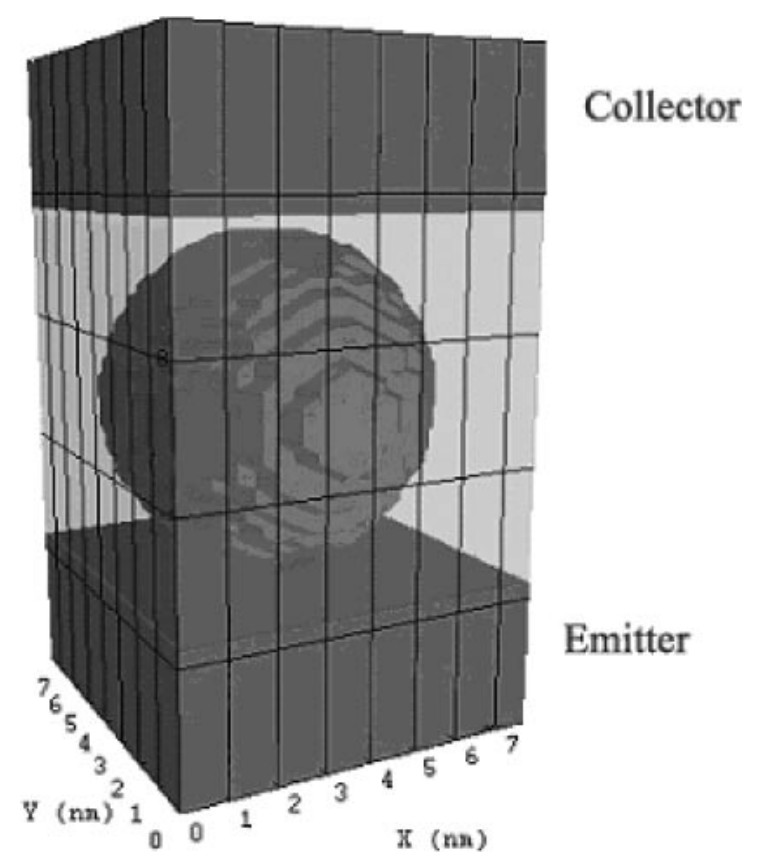

Fig. 4. 3D Structure of nc-Si QD sandwiched between two oxide barriers used for simulation.

incident mode taken at the first energy peak is shown in Fig. 6. The orthoslices are taken at the emitter, poles of the sphere, and the center of the sphere, all of which show the spherical s-orbital-like nature of the first quasi-bound state of the nc-Si dot. A p-orbital-like nature is seen for the quasibound state in the nc-Si dot for the second incident mode taken at the second incident peak shown in Fig. 7. The 3D

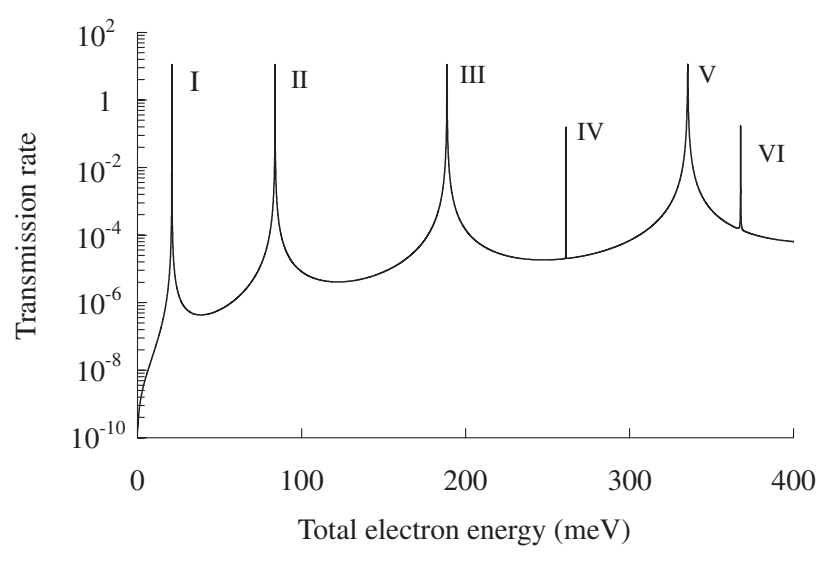

Fig. 5. Calculated transmission rates for single nc-Si QD RTD with $7 \mathrm{~nm}$ dot diameter. The first six energy peaks are identified.

probability density of electrons this time change from the first incident mode in the sphere pole to the second incident mode at the center of the sphere. Strong wave function localization occurs in the dot with the electron density occupying the volume of the QD.

We also studied the total tunnel current using the calculated transmission spectrum. We adopted a fine energy mesh of $0.02 \mathrm{meV}$ for the transmission calculations in order to obtain the total tunneling current precisely. The calculated $I-V$ characteristic is shown in Fig. 8. The bias voltage step here was chosen to be $0.5 \mathrm{mV}$.

In Fig. 8(a), we show the $I-V$ characteristics simulated at temperatures of $17 \mathrm{~K}, 77 \mathrm{~K}$, and room temperature. It was found that the $I-V$ curve at $17 \mathrm{~K}$ reflects the transmission

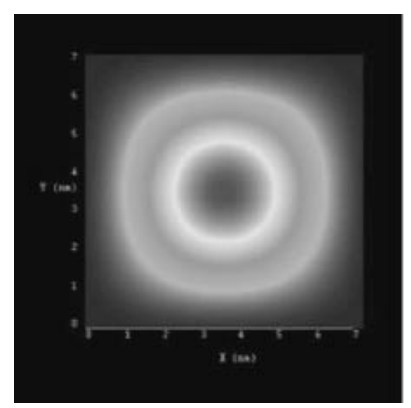

Emitter

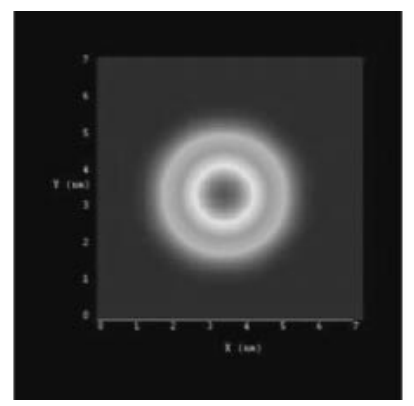

Dot, emitter pole

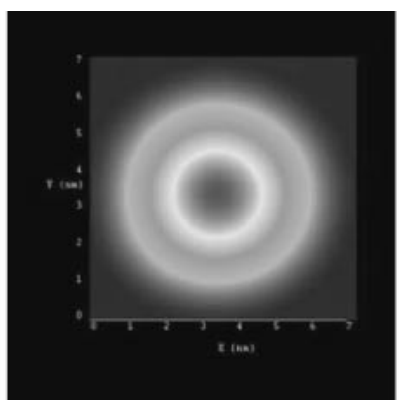

Center of dot

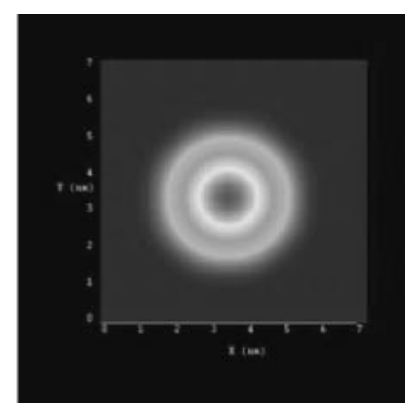

Dot, collector pole

Fig. 6. 3D probability density of electrons calculated for first-mode incident wave at first energy peak.

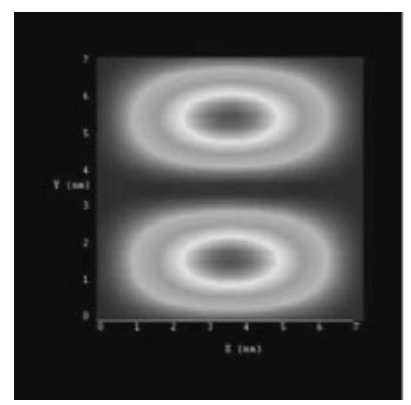

Emitter

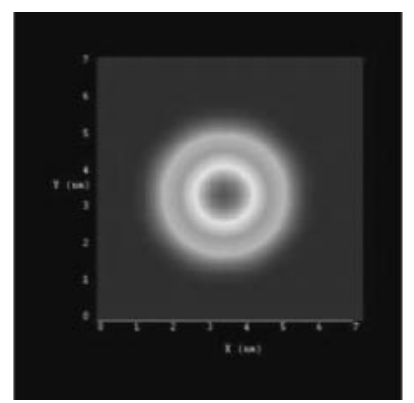

Dot, emitter pole

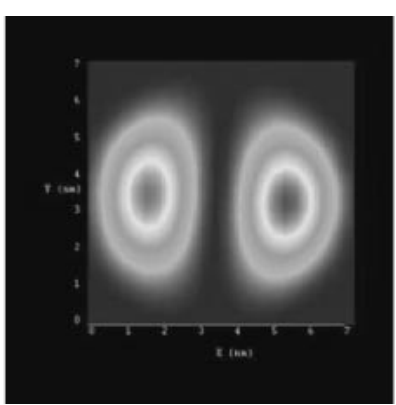

Center of dot

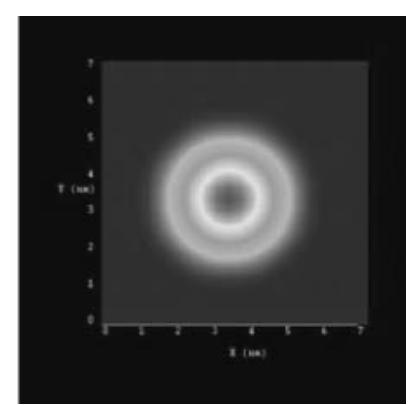

Dot, collector pole

Fig. 7. 3D probability density of electrons calculated for second-mode incident wave at second energy peak. 


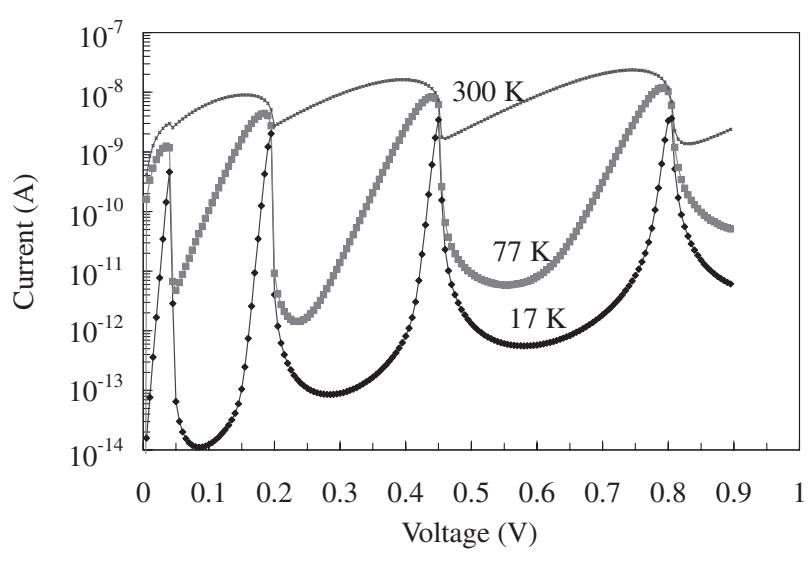

(a)

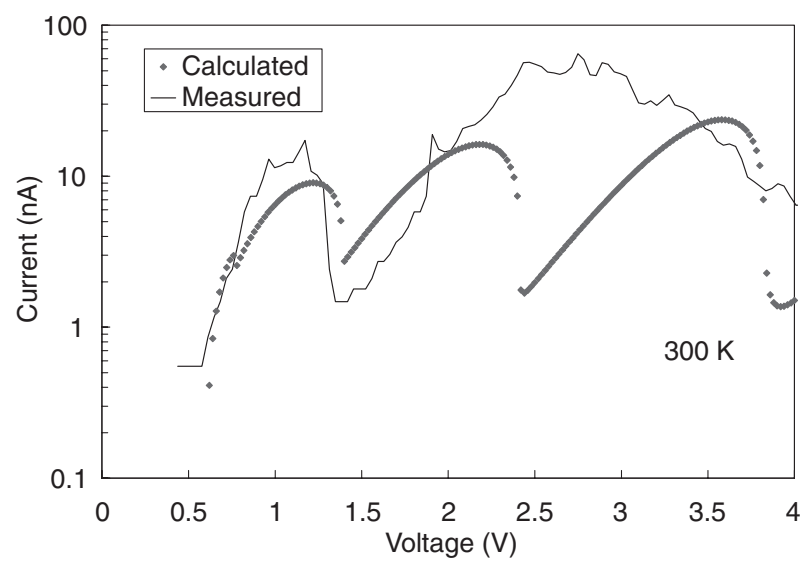

(b)

Fig. 8. Calculated $I-V$ characteristics (a) Original data from simulation at 17, 77, and $300 \mathrm{~K}$. (b) Comparison between experimental results and calibrated simulation results.

spectrum in Fig. 5 straightforwardly. Four current peaks result from the transmission peaks indicated by I, II, II and V in Fig. 5, respectively. Note that a sharp and low subpeak indicated by IV does not give a visible contribution to the $I-$ $V$ curve. At higher temperatures, the width of the individual current peaks increases markedly due to thermal broadening, resulting in a large increase in the valley currents. It should also be noted that the first current peak at room temperature is seen as simply a small shoulder on the second current peak. This is because the contributions from the first two transmission peaks (indicated by I and II in Fig. 5) overlap each other, and the two peaks merge virtually into one current peak.

By comparing these results with the experimental curve in Figs. 3(b) and 3(c), it is apparent that the observed peak voltages are much larger than simulated ones. One of the causes is the Schottky barrier formed between the gold AFM tip and $\mathrm{Si}$ with a height of about $0.7 \mathrm{eV}$. In addition, we suppose that the voltage drop across the nc-Si dot is only a fraction of the voltage applied between the AFM tip and the substrate. In Fig. 8(b), we tried to reproduce the experimental result by assuming that $25 \%$ of the total bias is applied to the nc-Si QD and the rest of the applied bias drops externally due to various factors such as contamination in the tip-dot interface. This ratio was determined so that the calculated peak voltage of the second current peak (first peak is merged into the second one as explained above) fits approximately to the observed first peak voltage in Fig. 3(b).

However, this result exhibits a large discrepancy regarding the observed large second current peak. The calculated $I-V$ curve does not give a high and broad peak as observed in the experiments. The widths of the current peaks at resonance are primarily determined by the intrinsic broadening of the individual transmission peaks, and therefore there is no way to calibrate the width of the second peak while that of the first peak is maintained. These results indicate that there is a likelihood that the first current peak is caused by resonant tunneling, whereas the second current peak is certainly not caused by it. The increase in the current after the first current peak is attributable to the background current which is commonly observed in conventional RTDs and is not included in our present simulation. We also learned from our past observations that the nc-Si QDs may move during the measurement with the high bias voltage applied to the tip. This causes a misalignment of the nc-Si dot and tip and eventually their separation. It is therefore supposed that the current drop after $2.5 \mathrm{~V}$ in Fig. 3(b) is due to such displacement of the nc-Si QD, and this scenario is also consistent with the large current fluctuation observed over the second current peak. Further careful investigations are obviously needed for the large-bias regime to probe this hypothesis.

\section{Conclusions}

We have observed the tunneling properties of a single spherical nc-Si quantum dot at room temperature for the first time by contact-mode AFM. A current peak has been observed with the highest peak-to-valley current ratio of about 17 , which may be associated with resonant tunneling via the single nc-Si quantum dot. We have also calculated the transmission spectra and associated tunneling current on the basis of the $3 \mathrm{D}$ scattering matrix theory. By using the simulations we have demonstrated resonant tunneling through the quasi-bound states formed in a single spherical silicon quantum dot.

\section{Acknowledgement}

The authors would like thank K. Usami for the nc-Si dot deposition, and Y. Tsuchiya and S. Huang for discussions.

1) R. Tsu and L. Esaki: Appl. Phys. Lett. 22 (1973) 562.

2) J. P. A. van der Wagt: Nanotechnology 10 (1999) 174.

3) J. P. A. van der Wagt, A. C. Seabaugh and E. A. Beam, III: IEEE Electron Device Lett. 19 (1998) 7.

4) A. C. Seabaugh, Y. C. Kao, J. Randall, W. Frensley and A. Khatibzadeh: Jpn. J. Appl. Phys. 30 (1991) 921.

5) Y. Ishikawa, T. Ishihara, M. Iwasaki and M. Tabe: Electron. Lett. 37 (2001) 1200

6) M. Fukuda, K. Nakagawa, S. Miyazaki and M. Hirose: Appl. Phys. Lett. 70 (1997) 2291.

7) J. Randall, G. Frazier, A. Seabaugh and T. Boerkaert: Microelectron. Eng. 32 (1996) 15.

8) J. Berg, S. Bengtsson and P. Lundgren: Solid-State Electron. 44 (2000) 2247.

9) H. Mizuta and T. Tanoue: The Physics and Applications of Resonant Tunneling Diodes (Cambridge University Press, Cambridge, 1995) Chap. 6, p. 185.

10) M. Mendoza and P. A. Schulz: Phys. Rev. B 68 (2003) 205302. 